\title{
A THz Superconducting Imaging Array Developed for the DATE5 Telescope
}

\author{
Sheng-Cai Shi ${ }^{1,2}$. Wen Zhang ${ }^{1,2} \cdot \operatorname{Jing} \mathrm{Li}^{1,2}$. \\ Wei Miao ${ }^{1,2}$ Z Zhen-Hui Lin ${ }^{1,2}$ - Zheng Lou ${ }^{1,2}$. \\ Qi-Jun Yao ${ }^{1,2}$
}

Received: 24 September 2015 / Accepted: 22 October 2015 / Published online: 6 November 2015

(C) The Author(s) 2015. This article is published with open access at Springerlink.com

\begin{abstract}
Dome A in Antarctica, located at an altitude of $4093 \mathrm{~m}$ and with very low temperature in winter down to $-83^{\circ} \mathrm{C}$, is an exceptionally dry site. Measurements of the atmospheric transmission in the range of $0.75-15 \mathrm{THz}$ by a Far-infrared/THz Fourier transform spectrometer (FTS) strongly suggest that Dome A is a unique site for ground-based THz observations, especially for the 200- and 350-micron windows. A 5-m THz telescope (DATE5) is therefore proposed for Chinese Antarctic Kunlun Observatory. We are currently developing a $\mathrm{THz}$ superconducting imaging array (TeSIA) for the DATE5. The TeSIA will be working at the $350-\mu \mathrm{m}$ window, with a pixel number of $32 \times 32$ and a sensitivity $(\mathrm{NEP})$ of $\sim 10^{-16} \mathrm{~W} / \mathrm{Hz}^{0.5}$. Ti transition-edge sensors with time-domain multiplexing and TiN microwave kinetic inductance detectors with frequency-domain multiplexing are both developed for the TeSIA. In this paper, detailed system designs and some measurement results will be presented.
\end{abstract}

Keywords Terahertz $\cdot$ TES $\cdot$ MKIDs $\cdot$ TeSIA $\cdot$ DATE5 $\cdot$ Dome A

\section{Introduction}

At THz and Far-infrared (FIR) wavelengths, we can observe early distant objects, most cold objects emitting the majority of their light, and obscured objects invisible in the optical due to the presence of dust. In addition, it is a frequency regime where there is an abundance of molecular spectral lines and fine atomic structure spectral lines

\footnotetext{
$凶 \quad$ Sheng-Cai Shi

scshi@pmo.ac.cn

1 Purple Mountain Observatory, CAS, Nanjing, China

2 Key Laboratory of Radio Astronomy, CAS, Nanjing, China
} 
which are very important tracers for probing the physical and chemical properties and dynamic processes of objects such as stars and planetary systems. As is well known, however, water vapor renders the terrestrial atmosphere opaque at THz and FIR wavelengths. This has driven large ground-based millimeter and submillimeter astronomical observatories to successively higher and drier sites. Dome A, the highest point of the Antarctic ice sheet, is an exceptionally dry site because of high altitude (4093 m) and low temperature (with a record of $-83^{\circ} \mathrm{C}$ ). Radiometric measurements at $0.66 \mathrm{THz}$ at Dome A have found that typical perceptible water vapor (PWV) in summer is only about $0.14 \mathrm{~mm}[1,2]$. Our recent FTS measurements demonstrate promising results at $\mathrm{THz}$ wavelengths [3]. Moreover, Dome A is an excellent year-round observing site in the submillimeter windows below $1 \mathrm{THz}$. Dome $\mathrm{A}$ is therefore a unique site for ground-based $\mathrm{THz}$ observations, especially for the $200-$ and $350-\mu \mathrm{m}$ windows. China is planning to build an observatory there, namely Chinese Antarctic Kunlun Observatory, with two proposed scientific facilities including a 5-m $\mathrm{THz}$ telescope named DATE5 [4].

A science case for the DATE5 telescope is to observe extreme starburst galaxies at different red-shifts to better understand the nature and the evolution of these enigmatic and important objects. To meet this requirement, we are developing a superconducting imaging camera (TeSIA) [5], which has an array size of $32 \times 32$ pixels and requires a background-limited sensitivity as high as $10^{-16} \mathrm{~W} / \mathrm{Hz}^{0.5}$ at $350 \mu \mathrm{m}$ (centered at approximately $0.85 \mathrm{THz}$ ). Note that the background-limited sensitivity is estimated for a $10 \%$ relative bandwidth and $50 \%$ atmospheric transmission at $350 \mu \mathrm{m}$. Ti transition-edge sensors (TES [6]) with time-domain multiplexing and TiN microwave kinetic inductance detectors (MKIDs [7]) with frequency-domain multiplexing are both adopted for the TeSIA development. A long-wavelength $(850 \mu \mathrm{m}$ or $0.35 \mathrm{THz}$ ) prototype with a smaller size of $8 \times 8$ pixels will be firstly installed on the portable submillimeter telescope (POST [8]) located in western China for demonstration.

\section{TES Developments for TeSIA}

Fig. 1 shows the schematic of the TeSIA developed with TES. The $32 \times 32$ TES detector array, operated at $300 \mathrm{mK}$, is indeed composed of four tiles of $16 \times 16 \mathrm{Ti}$ TES detectors. Time-domain multiplexing (TDM) is adopted for the readout of the TES detectors. There are eight readout channels in total, with each including 128 SQUIDs at $1 \mathrm{~K}$, an ASIC at $4 \mathrm{~K}$, an SR560 low-noise preamplifier, and a DAQ. SQUIDs used here are developed by STAR Cryoelectronics, with a critical current $2 I_{c}$ of $26 \mu \mathrm{A}$, an input inductance of $650 \mathrm{nH}$, and current noise lower than $1 \mathrm{pA} / \mathrm{Hz}^{0.5}$. The ASIC, designed by the Laboratoire AstroParticule et Cosmologie, Université Paris-7, is based on AMS 350-nm BiCMOS SiGe technology. A multiplexing factor of 128 is realized through the use of two levels of multiplexing. Details of the TDM readout are described in [9].

TES detectors adopted here are a single Ti microbridge acting as both absorber and thermometer, which is similar to that proposed by Karasik et al. [10]. As Ti superconducting films of a few tens of nanometers have a critical transition temperature 


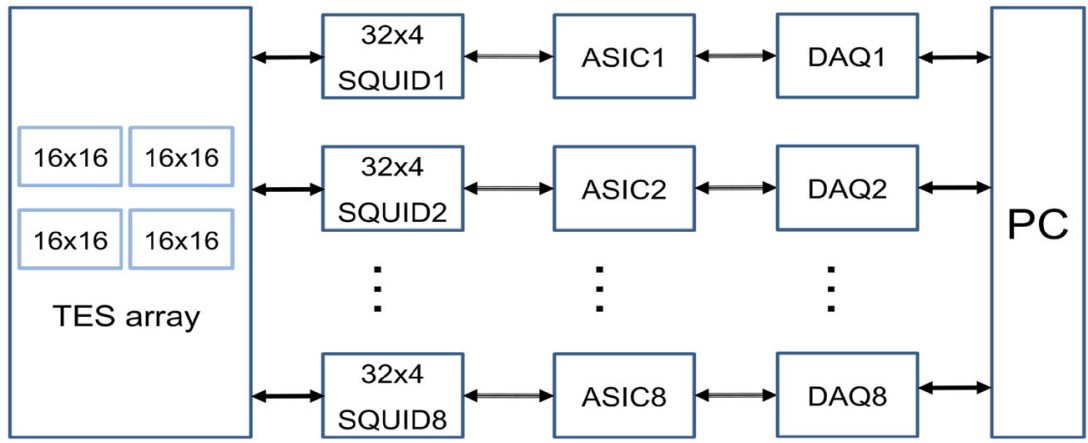

Fig. 1 Schematic of the TeSIA developed with Ti TES. The optics is not shown here (Color figure online)
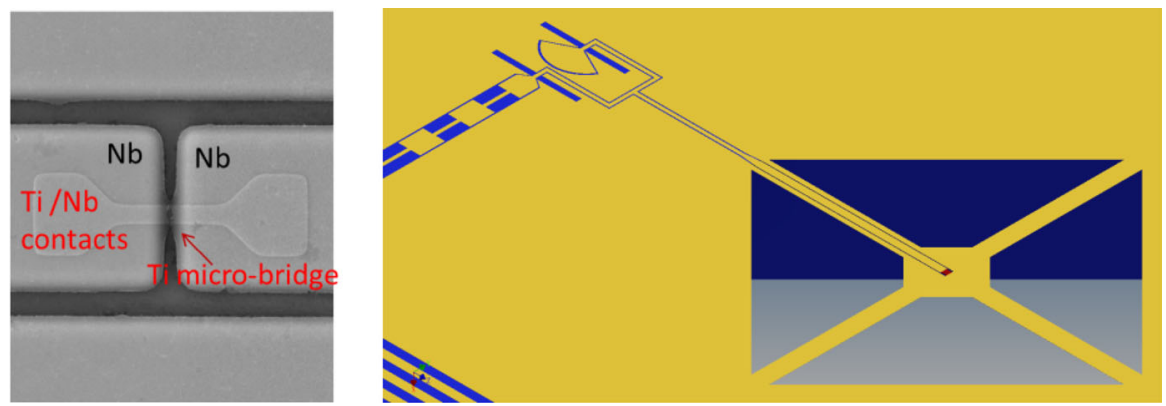

Fig. 2 Scanning-electron-microscopy (SEM) photo of a fabricated Ti TES (left) and schematic of a Ti TES with improved design with four SiN/Nb legs supporting the TES (right) (Color figure online)

around $400 \mathrm{mK}$, they are just suitable for the operating temperature of our detect array. The electric NEP of fabricated Ti TES detectors, as shown in Fig. 2, has reached $4 \times 10^{-17} \mathrm{~W} / \mathrm{Hz}^{0.5}$, which is close to the phonon noise-dominated NEP [11]. The measured effective time constant, however, is about $3 \mu \mathrm{s}$, which is still too fast for our TDM readout. Using an electron-phonon decoupled Ti TES, as shown in Fig. 2, can increase the time constant up to ms. As can be seen in Fig. 2 (right), the TES is supported by four $\mathrm{SiN} / \mathrm{Nb}$ legs, while the signal is coupled from a twin-slot antenna through a CPW transmission line.

The twin-slot antennae are coupled to the telescope relay optics by a silicon microlens array. The microlenses are hyper-hemispherical lenses with a diameter of $1.5 \mathrm{~mm}$ and pixel spacing of $\sim 1.9 \mathrm{~F} \lambda$ at $350 \mu \mathrm{m}$. The telescope relay optics include an ellipsoidal mirror (M5), two planar mirrors (M6 and M7), and a cold silicon lens (L1), which together transform the F/17 beams from the telescope coude focus to the final telecentric beams of F/2.3, maximizing the coupling to the microlenses. A diffraction-limited field of view of greater than 12 arcmin is achieved, satisfying the field requirement by the $32 \times 32$ TES or MKID array. The schematic of the optics is shown in Fig. 3. 
Fig. 3 Schematic of the designed optics for the TeSIA on the DATE5 telescope (Color figure online)
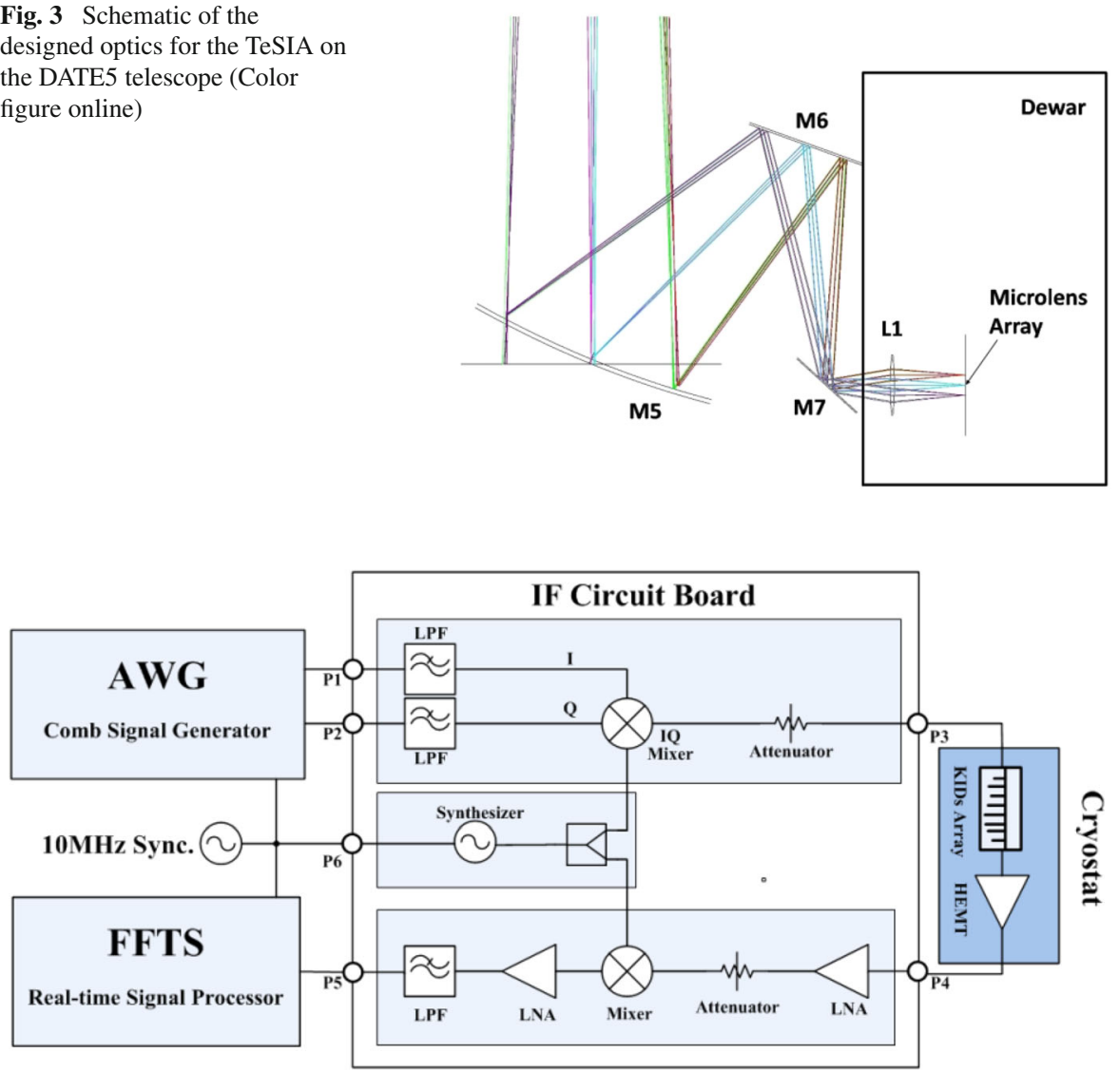

Fig. 4 Schematic of the TeSIA developed with antenna-coupled TiN MKIDs. The optics is not shown here (Color figure online)

\section{MKIDs Developments for TeSIA}

Fig. 4 shows the schematic of the TeSIA developed with MKIDs. The $32 \times 32$ MKIDs array adopts quarter-wavelength coplanar-waveguide (CPW)-type resonators based on 80-nm-thick TiN superconducting films with a critical temperature of approximately $4.5 \mathrm{~K}$ [12]. Such a superconducting film is just suitable for both the operating temperature of $0.3 \mathrm{~K}$ as well as the operating frequency of $0.85 \mathrm{THz}$. The $32 \times 32$ MKIDs are coupled to twin-slot antennae designed at $350 \mu \mathrm{m}$. Their resonance frequencies are distributed in the frequency range of $4-7.069 \mathrm{GHz}$ with an interval of $3 \mathrm{MHz}$. The designed coupling Q factors range from 50 to $1000 \mathrm{k}$. The FDM readout for this MKIDs array is similar to those used by other groups, but adopting a commercial arbitrary wave-function generator to generate 1024-tone input signal of 30-dB SNR.

The design and fabrication of the $32 \times 32$ MKIDs operating at $350 \mu \mathrm{m}$ follows that described in [12]. The estimated generation-recombination NEP is in the order 
of $10^{-16} \mathrm{~W} / \mathrm{Hz}^{0.5}$ for the operating temperature of $300 \mathrm{mK}$. The optics for the TeSIA developed with MKIDs is the same as that with TES, just as shown in Fig. 3.

\section{Conclusion}

We are developing a TeSIA for the DATE5 telescope to be constructed at Dome A in Antarctic. Ti transition-edge sensors (TES) with time-domain multiplexing and TiN microwave kinetic inductance detectors (MKIDs) with frequency-domain multiplexing are adopted for the TeSIA development. A long-wavelength $(850 \mu \mathrm{m}$ or $0.35 \mathrm{THz})$ prototype with a smaller size of $8 \times 8$ pixels has demonstrated good performance, and this will be installed on the POST located in western China for demonstration. Regarding the choice between TES and MKIDs for the DATE5 telescope, we will make an evaluation after the characterization of the $32 \times 32$ TES and MKIDs arrays, mainly in terms of sensitivity, easiness of remote operation, and power consumption.

Acknowledgments This work was supported in part by the National Natural Science Foundation of China (NSFC) under Grant No 11127903 and Chinese Academy of Sciences (CAS) under the Strategic Priority Research Program XDB04010300. We are grateful to the team led by M. Piat at APC (France) and F. Pajot at IAS for the development of TDM readout and the team led by C. Otani at RIKEN (Japan) for their assistance in TiN MKIDs fabrication.

Open Access This article is distributed under the terms of the Creative Commons Attribution 4.0 International License (http://creativecommons.org/licenses/by/4.0/), which permits unrestricted use, distribution, and reproduction in any medium, provided you give appropriate credit to the original author(s) and the source, provide a link to the Creative Commons license, and indicate if changes were made.

\section{References}

1. H.G. Yang et al., PASP 121, 174 (2009)

2. R. Stone, Science 329, 1136 (2010)

3. S.C. Shi et al in preparation

4. J. Yang et al., Res. Astron. Astrophys. 13, 1493 (2013)

5. S.C. Shi et al. ISSTT2014 (2014)

6. K.D. Irwin, G.C. Hilton, Cryogenic Particle Detection, Topics in Applied Physics 99 (Springer, Berlin, 2005)

7. Peter K. Day et al., Nature 425, 817 (2003)

8. J. Li et al., Appl. Phys. Lett. 92, 222504 (2008)

9. D. Prele et al., J. Low Temp. Phys. 176, 433 (2014)

10. B.S. Karasik et al., Appl. Phys. Lett. 98, 193503 (2011)

11. W. Zhang et al. in this Special Issue of J. Low Temp. Phys

12. J. Li et al. in this Special Issue of J. Low Temp. Phys 